\title{
Psychological Practice and the COVID-19 Crisis: A Rapid Response Survey
}

\author{
Morgan T. Sammons • Gary R. VandenBos • Jana N. Martin \\ Published online: 8 May 2020 \\ (C) National Register of Health Service Psychologists 2020
}

\begin{abstract}
Psychological practice has changed dramatically over the past 125 years. The two world wars both served to stimulate and change the scope of practice for psychologists. We surveyed over 3,000 doctoral psychologists about the impact of the COVID-19 health crisis on their clinical practices. Practice changed from primarily in-office to mostly telepsychology practice over the course of 2 weeks in March of 2020. The long-term effect on professional practice in psychology is not known.
\end{abstract}

\section{A Short Historical Note About Clinical Psychology}

The first psychological clinic was established in 1896 by Lightner Witmer in Philadelphia. For the next 20 years, the number of practicing psychologists remained small, and service settings were highly varied-university lab/clinics, public clinics/ hospitals, and a few in independent practice. The first major change in the magnitude, location, and nature of practice occurred during 1914-1918 with World War I and the need to evaluate new recruits for potential military service, particularly with the deployment of the Army Alpha and Beta tests. Clinical psychology and clinical practice experienced a big growth in psychological testing (particularly IQ testing). Growth and range of practice in the 20 years after the war were modest, but not insignificant (VandenBos, Cummings, \& DeLeon, 1992).

The second major change in the magnitude, location, and nature of practice occurred between 1939 and 1945. World War II and the need to quickly rehabilitate soldiers injured in battle or who were suffering psychological breakdown in combat spurred the onset of psychological interventions (Sammons, 2006).

After the war, the treatment of returning veterans was a major national priority, and the creation of the modern Veteran's Administration (VA; and the university-based clinical psychology training programs needed to support the VA psychological services) followed. The establishment of the National Institute for Mental Health at the end of the 1940s and its mental health training programs supported program training of clinical psychologists in the U.S., as it continues to do today. Both these efforts spurred the need for clinical psychologists, as well as increased diversity of psychological services throughout the country.
Clinical psychology, as a field and as a practice, has continued to grow organically over the past 70 years. Although several notable events contributed to the growth, no single major event contributed to the magnitude of change to the practice of psychology like the two major wars (Sammons, 2005). The COVID-19 health crisis in early 2020 may, however, have an influence not unlike that of the two world wars, if the results of the survey we report here persist over time.

\section{The Emerging and Evolving World of Telehealth}

Electronic forms of communication have a long history in medicine and psychology. An 1879 article in the Lancet discussed using the telephone to reduce unnecessary office visits. Beginning in the 1920 s, radio transmissions were used to give medical advice to clinics on ships at sea, and now applications of telemedicine, including robotic surgery, are plentiful (Nesbitt, 2012).

Meaningful experimentation with telehealth did not begin until the 1960s. In 1964, the Nebraska Psychiatric Institute began using television links to form two-way communication with the Norfolk State Hospital, 112 miles away, for education and consultation purposes. In 1967, Massachusetts General Hospital established an in-house medical station at Logan International Airport in Boston to provide health care for travelers via combined microwave audio and video links. This more or less coincided with NASA's foray into telehealth through the use of physiologic monitors for astronauts (Nesbitt, 2012). Alaska has been a model for the development and use of telehealth for decades because of the need to provide care to remote and hard-to-reach areas, 
likewise Hawai'i and telehealth connections with outlying Pacific islands.

Other telehealth projects were underway before and during the 1980s, but many of these failed to enter mainstream health care. Federal and state funding decreased, but individual hospitals began to launch their own telehealth initiatives. Following the 1985 Mexico City earthquake, NASA provided a satellite to enable health care communications of American Red Cross and Pan American Health Organization response teams. Florida first experimented with telehealth in its prisons during the latter 1980s.

The advent of high-speed internet and increased access to wireless laptops and mobile phones made telehealth more plausible by the mid-1990s. The first use of "telepsychology" in the abstract of a journal covered in APA PsycInfo was of an article by Stiers and Kewman (1997). The first use of the term "telepsychology" in an article title of an APA journal occurred in a special issue of Professional Psychology in 2000. One of those articles was a survey of 596 practicing psychologists about the nature and extent of psychologists' involvement in telepsychology (VandenBos \& Williams, 2000), reporting that only $2 \%$ had ever used internet or satellite technology to deliver health care. In 2019, a survey of 1,791 psychologists averaging 26 years of experience found that the vast majority of psychologists had still never used telepsychology (Pierce, Perrin, \& McDonald, 2020).

The passage of the Patient Protection and Affordable Care Act (Obamacare) in March of 2010 triggered rapid growth in telehealth equipment, software, and electronic health record systems. Reviewing the randomized controlled trial literature from 2005 through 2016 regarding the telepsychological treatment of four mental health conditions (depression, anxiety, PTSD, and adjustment disorders) found evidence to support video conferencing and teleconferencing interventions (Varker, Brand, Ward, Terhaag, \& Phelps, 2019). Campbell, Millan, and Martin (2018) in a series of case studies explored processes for informed consent in telepsychology, an important new practice development. Psychological practice experienced massive change in March of 2020, resulting from the COVID-19 health crisis.

\section{Method}

We wanted to learn more about psychologists' prior telepsychology experiences, the current use of telepsychology since the onset of the COVID-19 crisis, and the nature of the psychological services currently being provided. The Trust and the National Register each sent a brief email survey to Trust Insureds and members of the National Register on March 26, 2020, six days after the secretary of the U.S. Department of Health and Human Services (n.d.) declared COVID-19 to be a national public health emergency and approximately 2 weeks after significant public health measures against COVID-19 had begun. The survey remained open for 12 days, until April 6, 2020. One reminder was sent.

We asked about psychologists' prior telepsychology experiences, the use of telepsychology since the onset of the COVID-19 crisis, and the nature of the psychological services currently being provided. We developed a brief questionnaire with 19 multiple- or forced-choice questions and one openended question (see Appendix). We asked about years of professional experience and employment setting, as it seemed these two factors might show the biggest differences. We asked about prior, current, and future uses of telepsychology delivery platforms. And we asked about the impact of the COVID-19 crisis on caseload, as well as patient satisfaction with online psychological care.

The Trust and the National Register sent email invitations to their respective practitioner groups of licensed, doctorallevel psychologists. Over 22,000 psychologists $(\mathrm{n}=22,392)$ opened the email invitation. Of these, 3,038 returned the survey, representing an overall response rate of $13.6 \%$. This is an acceptable number for a rapid-response email survey of short duration; however, generalizability concerns remain and caution should be used in interpreting these data. In our analysis, responses are combined for both organizations unless stated otherwise. Because not all respondents answered all questions, individual $n$ s for specific queries are noted as appropriate.

We divided respondents according to years of licensure, with groupings for those licensed from $1-5,6-10,11-15$, $16-20,21-25$, and over 26 years postlicensure. Respondents tended to be more senior psychologists; $46 \%$ of respondents reported being licensed for 26 years or more, with the remainder being equally distributed among those licensed for 5,10 , 15,20 , or 25 years.

\section{Results}

Mindful that work setting strongly dictates both individual and institutional responses to the COVID-19 crisis, we queried respondents ( $n=3,015$ usable responses) regarding their primary work setting. Seventy-four percent of respondents reported solo or group private practice work settings, while $23 \%$ were employed in hospitals, clinics, agencies, or other institutional settings.

Variance according to the number of years postlicensure was noted. Psychologists licensed for more than 26 years accounted for half (51\%) of all those working in solo or group private practices. In contrast, psychologists licensed for 15 years or fewer were more likely to report primary employment in an agency, clinic, or organized health care setting. This aligns with broad employment trends of early and midcareer psychologists practicing in institutional settings. 
Of significant concern is that most respondents reported a decrease in caseload since the inception of the COVID-19 crisis. Of the 2,997 responses to this question, 59\% reported a decline in caseload. One third of respondents (34\%) reported a stable caseload, and a small minority (6\%) reported an increase in caseload. This will have significant repercussions on psychologists' income, especially for those in solo or group private practices.

We queried respondents about how the COVID-19 crisis has affected their use of telepsychology services, defined as fully integrated audio and video capability, textbased services (chat or email communication), or telephone. Categories were not exclusive. A dramatic shift in the use of telepsychology was reported. Prior to the COVID-19 crisis, only 29\% reported using any form of telepsychology at some times for some services, but after the onset of the crisis, $83 \%$ of respondents reported using it almost exclusively. The vast majority of the subset not using telepsychology at the time of the survey (73\%) reported their intention to do so in the near future.

Respondents mostly provided services to established patients and did not perform initial intakes via telepsychology at this point (just a couple of weeks into the COVID-19 crisis). Seventy percent of respondents saw established patients, only $30 \%$ had performed new clinical intakes. Psychologists who were more recently licensed were more likely to conduct initial intakes using distance modalities; $41 \%$ of those licensed for fewer than 5 years performed such intakes, as did $37 \%$ of those licensed for 6-10 years. Only $26 \%$ of those licensed for more than 26 years performed distance intakes so far.

Despite practitioners' rapid adoption of telepsychology platforms, patient reactions to receiving telepsychology services (as estimated by our respondents) were decidedly mixed. We asked respondents to identify the percentage of their patients whom they believed liked, disliked, or had neutral feelings toward receiving telepsychology services. Only $20 \%$ of respondents reported that the plurality of their patients liked telepsychology services. Most respondents (86\%) reported that as many as half of their patients did not like such services. On the other hand, responses also indicated that many patients remained neutral, at least in the psychologists' perception, about such services, with $90 \%$ of respondents reporting that a majority of their patients were neutral on the subject.

The rapid shift to distance service provision may have been facilitated by respondents' perceptions that they were relatively prepared to deliver telepsychology services. Fifty-eight percent of respondents reported feeling somewhat or extremely prepared to conduct telepsychology, opposed to only $20 \%$ who reported being extremely or somewhat unprepared. Duration of licensure did not affect levels of preparedness, with more senior psychologists (26 years or more postlicensure) reporting being as prepared or more prepared than those in younger cohorts.
Although respondents reported using a combination of distance service methods $(\mathrm{n}=2,627)$ combining telephone, video conferencing, and text-based (chat, messaging, email) methods, the largest percentage $(88 \%)$ provided at least some services using video conferencing, often in combination with telephone and text-based platforms. We did not query as to whether platforms were used for the provision of psychological services or for more general functions, such as scheduling and billing.

We queried respondents regarding the platforms used in providing telepsychology services ( $n=3,550$ as some respondents used more than one platform). Of the five most commonly used platforms, 29\% used Doxy.Me, 25\% used Zoom, 15\% used FaceTime, 7\% used Skype, and 4\% used Simple Practice. Other platforms such as VSee, WebEx, Thera-Link, and WhatsApp were endorsed by smaller numbers of respondents. Institution-specific platforms associated with electronic health records (e.g., EPIC) or the Veteran's Administration videoconferencing system were used by others.

We also included the following open-ended question: What professional information and/or tools do you need that are not currently available from the National Register or TrustPARMA (or other organizations)? Over 1,000 respondents provided commentary or requests. The top five mostfrequently mentioned topics were (a) how to do psychological testing online, (b) specific information and detail on billing and coding, (c) sample forms and recommendations on getting/receiving forms during the crisis, (d) recommendations about platforms (including features and security) to use for telepsychology, and (e) how to do online screening and intakes. Other requests included information on interstate practice, Medicare and insurance coverage for phone sessions, and training for conducting online supervision. The last group of notable requests included providing online services to children and youth, conducting group and family therapy online, and marketing/referral generation. Information on best practices (including ethics and risk management) for telepsychology was also requested. We are pleased to report that this content was provided in seven webinars hosted by the National Register and The Trust in April of 2020 (available at https://www.nationalregister.org/education-training/webinars; https://parma.trustinsurance.com/Resource-Center/COVID19-Resources).

\section{Discussion}

This brief, rapid response survey of licensed psychologists yielded some insight into how psychologists' practices have changed in the initial phases of the era of physical distancing mandated by the COVID-19 crisis.

Perhaps our most significant finding was the almost "overnight" transition from in-office practice to online 
practice. Telepsychology practice went from $29 \%$ of users employing online platforms for a portion of their practice to over $80 \%$ conducting almost all their practice online. This is an amazing practice transformation that occurred in just 1-2 weeks. This rapid shift occurred because the technology was available, and some processes and procedures were already in place for telepsychology, including online practice guidelines (Joint Task Force for the Development of Telepsychology Guidelines for Psychologists, 2013). Additionally, most psychologists had some level of exposure to telepsychology practice even if they were not doing it regularly.

Our second most significant finding was the $30 \%$ overall reduction in psychologists' caseloads. Obviously, nonsalaried psychologists in independent practice are most affected by this decline, but those in institutional settings may also feel job insecurity in that productivity measures such as relative value units are likely to decrease. The medium and long-term consequences of this caseload reduction are not yet apparent, but we believe that dues-driven psychological organizations may see a reduction in their overall memberships, and organizations that provide services to psychologists, such as meetings and conventions, may also experience reduced attendance numbers.

We remain concerned that psychologists may not have adequate information to buttress the belief that they are appropriately prepared to conduct telepsychology competently and with minimal risk to clients and themselves. From the inception of service delivery to evaluation of outcomes of assessment or psychotherapy conducted via telepsychology, fundamental practical and risk-management differences exist. Psychologists may be unaware that a separate informed consent form is required when transitioning from in-person to telepsychology services. An explicit safety plan including emergency contacts and resources at the patient's location is likewise required, as are protocols to ensure patient privacy when receiving at-home psychological services (Alvord, 2020).

Standardized assessment instruments have typically not been normed for remote administration. Visual clues that are apparent in an in-person evaluation may be lacking in remote consultations, depriving the psychologist of information that may inform diagnoses and treatment decisions. These among other factors make it problematic to conduct an initial intake via telepsychology.

The U.S. Department of Health and Human Services and the Centers for Medicare and Medicaid Services have temporarily waived enforcement protocols for violations of the HIPAA Privacy and Security Acts or breach notification rules for the duration of the COVID-19 crisis (U.S. Department of Health and Human Services, n.d.). Providers who are deemed to operate in good faith will not be prosecuted for privacy or security violations, regardless of whether or not the services delivered are specific to COVID-19.
This does not, however, render the psychologist immune from civil action resulting from inadvertent disclosure of protected health information, and individual jurisdictions may elect to pursue their own sanctions for such breaches. Also of concern is that some respondents used electronic service delivery platforms that may not have HIPAA required Business Associates Agreements (BAAs), such as WhatsApp. Although other platforms such as Doxy.Me or Zoom do provide BAAs, similar, less expensive platforms do not have privacy or security safeguards consistent with HIPAA standards. We did not query specifically as to BAAs associated with users' platforms. While it can be confusing and time-consuming to sift through the evolving changes, psychologists have an ethical and professional responsibility to ensure the protection of their client's welfare, which cannot be deferred. Consulting with others (always recommended even when there is no crisis) who have more experience practicing telepsychology or are aware of managing risk is critically important.

Changes in practice patterns in response to emergency situations occur by definition unpredictably and rapidly. Traditional methods for determining practice standards and ethical conduct of service provision during such times are insufficient. While preexisting guidance can provide a general framework for sound practice, such as the American Psychological Association's guidelines for the practice of telepsychology, practitioners must often rely on imperfect information to determine applicable standards. In such times, consultation and sound clinical judgment based on the needs of the individual patient may become the best sources of guidance for the practitioner. Also, documenting the thought and decision-making process is as important as the decision itself.

\section{Wrapping Up \& Reflection}

To recapitulate, the COVID-19 crisis has created a rapid shift in psychologists' practice patterns. In our survey, the vast majority transitioned very suddenly to online service delivery. Whether or not this phenomenon will persist after the crisis ends is unclear. Much depends on state and federal laws and regulations as well as reimbursement decisions made by the Centers for Medicare and Medicaid Services and private thirdparty payers. Ultimately, however, patient and provider acceptance will determine the extent to which distance service provision becomes a more widespread component of the treatment repertoire. It is our belief that, by availing themselves of extant and developing resources such as those provided by the National Register, The Trust, and other organizations, psychologists will become sufficiently comfortable with this medium to create lasting change, and that both practitioners and patients will benefit from expanded access to mental health services. 


\section{Appendix}

\section{Telepsychology in COVID-19 Survey}

We are conducting a brief survey to identify the needs of our psychologists during the COVID-19 pandemic. Your responses will help us understand how COVID-19 has affected your practice and your changing professional needs. The Trust and the National Register will use these responses to determine how best to help you meet the challenges of practice during this time.

The National Register and The Trust are committed to providing you with up-to-date risk management and clinical information. Additional resources on COVID-19 and telepsychology are available online (https://www. nationalregister.org and https://parma.trustinsurance.com).

1. How many years have you been licensed as a psychologist?
a. $0-5$
b. $6-10$
c. $11-15$
d. $16-20$
e. $21-25$
f. 26 or more

2. What is your primary employment setting?

a. Solo or group private practice

b. Agency, clinic, or organized health care setting

c. Primary or secondary school

d. Other

3. Were you conducting telepsychology before the COVID19 crisis?

a. Yes

b. No

4. Are you currently conducting telepsychology?

a. Yes

b. No

5. Are you planning to conduct telepsychology in the near future?

a. Yes

b. No

6. What method(s) are you using to conduct telepsychology services? (Please check all that apply) a. Chat or instant messaging (text-based, with no audio or video)

b. Telephone or web call (audio only)

c. Video conferencing service (audio and video)

d. Other

7. What video conferencing platform(s) are you using to conduct telepsychology services? (Please check all that apply)
a. $8 \times 8$
b. FaceTime
c. Doxy.Me
d. Skype
e. Vsee
f. Thera-LINK
g. WebEx
h. Zoom
i. Other

8. How prepared do you feel to conduct telepsychology services?
a. 1 - Extremely unprepared
b. 2
c. 3
d. 4
e. 5 - Extremely prepared

9. What percentage of your typical caseload were you providing via telepsychology before COVID-19?
a. $0-24 \%$
b. $25-49 \%$
c. $50-74 \%$
d. $75-100 \%$

10. What percentage of your typical caseload are you currently providing via telepsychology: Right Now?
a. $0-24 \%$
b. $25-49 \%$
c. $50-74 \%$
d. $75-100 \%$

11. What percentage of your typical caseload will you be providing via telepsychology next week?
a. $0-24 \%$
b. $25-49 \%$
c. $50-74 \%$
d. $75-100 \%$ 
12. What percentage of your typical caseload will you be providing via telepsychology next year?
a. $0-24 \%$
b. $25-49 \%$
c. $50-74 \%$
d. $75-100 \%$

13. Since the COVID-19 crisis, my patient caseload has
a. Increased
b. Stayed the same
c. Decreased

14. What percentage of your patients/clients like receiving care via telepsychology?
a. $\quad 0-24 \%$
b. $25-49 \%$
c. $50-74 \%$
d. $75-100 \%$

15. What percentage of your patients/clients have neutral feelings about receiving care via telepsychology?
a. $0-24 \%$
b. $25-49 \%$
c. $50-74 \%$
d. $75-100 \%$

16. What percentage of your patients/clients dislike receiving care via telepsychology?
a. $\quad 0-24 \%$
b. $25-49 \%$
c. $50-74 \%$
d. $75-100 \%$

17. Are you performing intakes for new patients virtually?
a. Yes
b. No

18. How are your new patients completing intake forms?

a. Hard copy form that is mailed or faxed

b. Hard copy form that is filled out in person at my office

c. Hard copy form that is emailed to me

d. Online form with payment sent directly to me

e. Online form on third-party site, electronically sent to me (including EHR)

f. Other
19. If you are a supervisor, are you providing supervision virtually?

a. Yes

b. No

20. What professional information and/or tools do you need that are not currently available from the National Register or TrustPARMA (or other organizations)? (Open answer)

\section{References}

Alvord, Mary K. (2020). Pragmatics of telepsychology practice in the age of COVID-19 [Webinar]. The National Register. 20 March 2020, online access verified 9 April 2020 at https://ce. nationalregister.org/videos/pragmatics-of-telepsychology-practicein-the-age-of-covid-19-archived/

Campbell, L. F., Millan, F., \& Martin, J. N. (Eds.). (2018). A telepsychology casebook: Using technology ethically and effectively in your professional practice. Washington, DC: American Psychological Association.

Joint Task Force for the Development of Telepsychology Guidelines for Psychologists. (2013). Guidelines for the practice of telepsychology. American Psychologist, 68, 791-800. https://doi.org/10.1037/ a0035001

Nesbitt, T. S. (2012). The evolution of telehealth: Where have we been and where are we going? In Institute of Medicine, Board on Health Care Services, \& T. A Lustig (Eds.), The role of telehealth in an evolving health care environment: Workshop summary (pp. 11-16). Washington, DC: National Academies Press. https://doi.org/10. 17226/13466

Pierce, B. S., Perrin, P. B., \& McDonald, S. D. (2020). Demographic, organizational, and clinical practice predictors of U.S. psychologists' use of telepsychology. Professional Psychology, 51, 184189. https://doi.org/10.1037/pro0000267

Sammons, M. T. (2005). Psychology in the public sector: Addressing the psychological effects of combat in the U.S. Navy. American Psychologist, 60, 899-909.

Sammons, M. T. (2006). Navy clinical psychology: A distinguished past and vibrant future. In A. D. Mangelsdorff (Ed.), Psychology in the service of national security (pp. 141-152). Washington, DC: American Psychological Association.

Stiers, W. M., \& Kewman, D. G. (1997). Psychology and medical rehabilitation: Moving toward a consumer-driven health care system. Journal of Clinical Psychology in Medical Settings, 4, 167-179.

U.S. Department of Health and Human Services, Office for Civil Rights (n.d.). FAQs on telehealth and HIPAA during the COVID-19 nationwide public health emergency. Retrieved from https://www.hhs.gov/ sites/default/files/telehealth-faqs-508.pdf

VandenBos, G. R., \& Williams, S. (2000). The internet versus the telephone: What is telehealth anyway? Professional Psychology, 31, 490-492. https://doi.org/10.1037/0735-7028.31.5.490

VandenBos, G. R., Cummings, N. A., \& DeLeon, P. H. (1992). A century of psychotherapy: Economic and environmental influences. In D. K. Freedheim (Ed.), History of psychotherapy: A century of change (pp. 65-103). Washington, DC: American Psychological Association.

Varker, T., Brand, R. M., Ward, J., Terhaag, S., \& Phelps, A. (2019). Efficacy of synchronous telepsychology interventions for people 
with anxiety, depression, posttraumatic stress disorder, and adjustment disorder: A rapid evidence assessment. Psychological Services, 16, 621-635. https://doi.org/10.1037/ser0000239

Morgan T. Sammons, PhD, ABPP, is the executive officer of the National Register of Health Service Psychologists. He is a retired Navy captain and was formerly the U.S. Navy's specialty leader for clinical psychology.
Gary R. VandenBos, PhD, is the senior professional consultant at the National Register of Health Service Psychologists. He previously served as the publisher of the American Psychological Association for over 30 years. He is the coauthor of Psychotherapy with Schizophrenics: The Treatment of Choice.

Jana N. Martin, $\mathrm{PhD}$, is the CEO of The Trust. Previously, she was a cochair of the Joint Task Force for the Development of Telepsychology Guidelines for Psychologists and a coeditor of A Telepsychology Casebook: Using Technology Ethically and Effectively in Your Professional Practice. 